\title{
Dysregulation of signaling pathways and putative biomarkers in liver cancer stem cells (Review)
}

\author{
KAI SONG $^{1-3}$, JUNHUA WU ${ }^{3}$ and CHUNPING JIANG ${ }^{1,2}$ \\ ${ }^{1}$ Department of Hepatobiliary Surgery, The Affiliated Drum Tower Hospital, School of Medicine, Nanjing University; \\ ${ }^{2}$ Institute of Hepatobiliary Surgery, Nanjing University; ${ }^{3}$ School of Medicine, Nanjing University, Nanjing, Jiangsu, P.R. China
}

Received August 16, 2012; Accepted September 25, 2012

DOI: $10.3892 /$ or.2012.2082

\begin{abstract}
Hepatocellular carcinoma (HCC) is one of the most common tumors in the world. At present, the details of the mechanism responsible for HCC formation and maintenance remain unclear. However, the cancer stem cell (CSC) theory suggests that liver cancer stem cells (LCSCs) may be responsible for the biological characteristics of HCC. Dysregulation of signaling pathways, including transforming growth factor $\beta$ (TGF- $\beta$ ), Wnt, Notch and Hedgehog pathways, has been found to be involved in the process of hepatocarcinogenesis and is considered the key determinant of LCSC function. Numerous LCSC biomarkers have been identified including CD133, epithelial cell adhesion molecule (EpCAM), ABCG2 and CD90, which would contribute to the isolation of LCSCs.
\end{abstract}

\section{Contents}

1. Introduction

2. Cancer stem cells

3. Liver stem/progenitor cells and liver cancer stem cells

4. Signaling pathways and liver cancer stem cells

5. Markers for liver cancer stem cells

6. Discussion

\section{Introduction}

Liver cancer is one of the most common cancers worldwide and is a main cause of cancer-related death. There are many risk factors related to hepatocellular carcinoma (HCC), such as hepatitis $\mathrm{B}$ virus (HBV) infection, hepatitis $\mathrm{C}$ virus (HCV) infection, alcohol abuse, obesity-related fatty liver disease,

Correspondence to: Professor Chunping Jiang, Department of Hepatobiliary Surgery, The Affiliated Drum Tower Hospital, School of Medicine, Nanjing University, Nanjing, Jiangsu, P.R. China E-mail: chunpingjiang@yahoo.com.cn

Key words: hepatocellular carcinoma, cancer stem cell, liver cancer stem cell, signaling pathway, putative biomarker aflatoxin and various carcinogens (1-5). Effective treatments for localized HCC include partial liver resection, liver transplantation and local ablation, such as radiofrequency ablation (RFA), interstitial laser coagulation, percutaneous ethanol injection (PEI) and percutaneous acetic acid injection (PAI) (6-8). These treatments result in a cure for cancer only for early stage tumors. Systemic therapy is the conventional treatment for advanced HCC, but the outcomes are not satisfactory. Therefore, the mechanisms involved in the formation and progression of HCC require further investigation to discover more effective therapies for liver cancer.

Currently, the theory of a 'cancer stem cell' may partially explain the process of HCC formation. According to the theory, there is a rare population of stem-like cells in tumor tissue, called liver cancer stem cells (LCSCs), which are responsible for the self-renewal, malignant transformation, metastasis and chemoresistance of HCC. Dysregulation of signaling pathways, including the transforming growth factor $\beta$ (TGF- $\beta$ ), Wnt, Notch and Hedgehog pathways, has been found to be involved in the process of hepatocarcinogenesis. In order to isolate LCSCs from tumor tissue, biomarkers need to be defined. At present, several markers that identify LCSCs have been reported. These include CD133, epithelial cell adhesion molecule (EpCAM), ABCG2 and CD90.

\section{Cancer stem cells}

Currently, all of the cancer cells in a tumor are thought to be responsible for tumor growth. However, recently emerging evidence suggests that there is a rare population of stem-like cells in tumors that determine cancer characteristics. Reya et al (9) proposed a theory of cancer stem cells (CSCs). They stated that 'tumors may often originate from the transformation of normal stem cells, similar signaling pathways may regulate self-renewal in stem cells and cancer cells, and cancer cells may include 'cancer stem cells' - rare cells with indefinite potential for self-renewal that drive tumorigenesis'.

Bonnet and Dick (10) first reported the existence of CSCs in acute myeloid leukemia (AML), and CSCs have been subsequently found in some solid tumors. Al-Hajj et al (11) first successfully isolated CSCs from breast tumors. Many studies also demonstrated the presence of CSCs in prostate $(12,13)$, lung $(14,15)$, colon $(16,17)$, pancreatic $(18,19)$ and brain tumors $(20,21)$. 


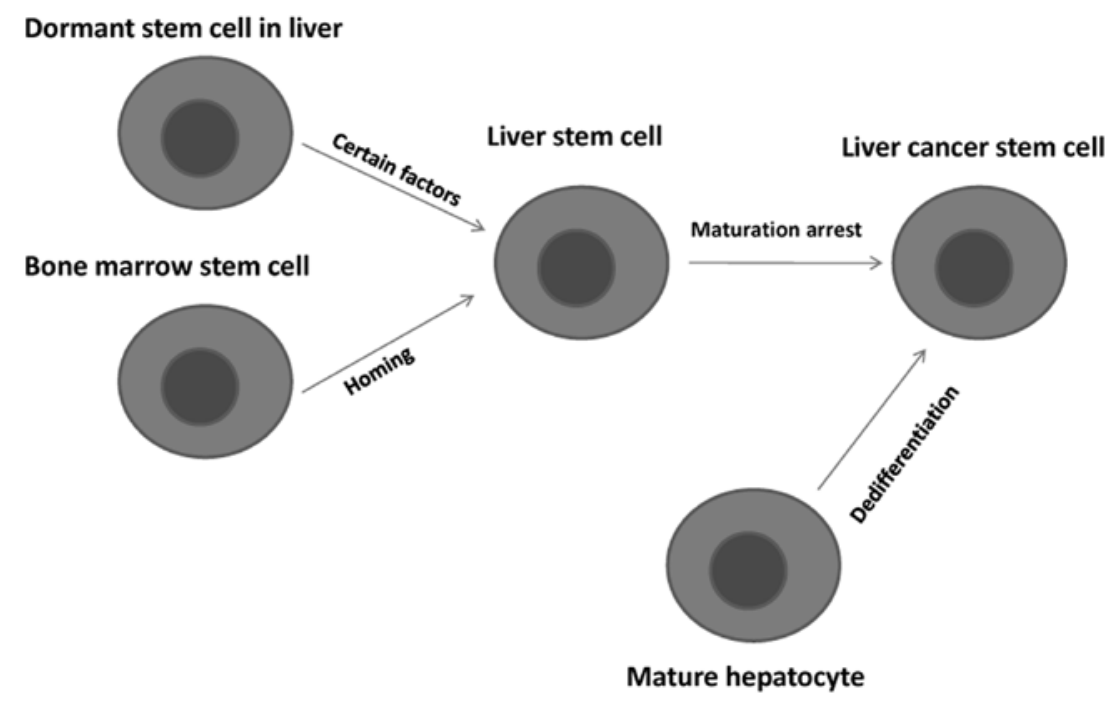

Figure 1. The origin of LCSCs. There are two main hypotheses that explain the origin of LCSCs: the dedifferentiation of mature hepatocytes and the maturation arrest of liver stem cells. Most studies currently support the latter one. There are two possible sources of liver stem cells, which are the reactivation of dormant stem cells in the mature liver by certain factors and the derivation of LCSCs from other organs, such as bone marrow.

At present, the mechanisms responsible for the formation and features of HCC are not clear, but the CSC theory suggests that LCSCs may be responsible for HCC. Sun et al (22) analyzed different expression patterns of stem-cell markers in HBV-associated cirrhotic livers and in HCC and demonstrated that the stem-like cells possessed tumorigenic capacity and that these cells might be LCSCs.

\section{Liver stem/progenitor cells and liver cancer stem cells}

Liver progenitor cells, a type of bipotential cell in human liver tissue, give rise to both hepatocytes and the biliary tree. There are two main potential sources of liver stem cells: adult liver stem/progenitor cells and extrahepatic stem cells. The adult stem cells reside in the mature liver and can be activated by certain factors. The oval cells, located in the canal of Hering, have the ability to differentiate into both hepatocytes and biliary epithelia and are now generally acknowledged to be liver stem/progenitor cells (23). In addition, liver stem cells may also be derived from other organs, such as bone marrow $(24,25)$. Increasing evidence shows that bone marrow stem cells participate in liver regeneration $(26,27)$ and that Thy1positive bone marrow stem cells might be the source of these liver stem cells (28).

The CSC theory suggests that LCSCs exist, but the origin of LCSCs is unclear. There are two main hypotheses to explain the origin of LCSCs: the dedifferentiation of mature hepatocytes and the maturation arrest of liver stem cells. Early studies in rat models mainly focused on premalignant foci and nodules, and the results supported the dedifferentiation hypothesis $(29,30)$.

However, this hypothesis has been challenged by subsequent research. At present, it is commonly believed that liver stem/progenitor cells are the potential source of HCC, intrahepatic cholangiocarcinoma (ICC), combined hepatocellular cholangiocarcinoma (CHC) and cholangiolocellular carcinoma (CLC), a subtype of cholangiocellular carcinoma (CC) (31-36). To study the effect of oval cells upon tumorigenesis, de Lima et al (37) established a rat model of non-alcoholic steatohepatitis (NASH), cirrhosis and HCC, showing that oval cells could proliferate in this model and that these cells may be the origin of malignancy. In another model (the Solt-Farber carcinogenic model), hepatic progenitor cells, identified by the expression of glypican-3 (GPC3), were shown to play an important role in hepatic carcinogenesis (38). A summary of the origin of LCSCs is presented in Fig. 1.

\section{Signaling pathways and liver cancer stem cells}

Dysregulation of signaling pathways has been observed in the process of hepatocarcinogenesis, and the TGF- $\beta$, Wnt, Notch and Hedgehog signaling pathways have been extensively studied. The signaling pathways involved in LCSCs are presented in Fig. 2.

TGF- $\beta$ signaling pathway. The TGF- $\beta$ signaling pathway plays a crucial role in cell cycle regulation, the immune system and apoptosis. In HCC, TGF- $\beta$ signaling inhibits oncogenesis at an early stage by inducing apoptosis (39). This physiological phenomenon is involved in TGF- $\beta$-induced TRAIL expression and in the ability of Smad 3 to repress Bcl-2 transcription and p53-dependent apoptosis, which is mediated by the TGF- $\beta$ signaling pathway (40-42). In addition, a recent study demonstrated that TGF- $\beta$ activates autophagy in certain HCCs to suppress tumor formation (43), and emerging evidence also suggests that dysregulation of TGF- $\beta$ signaling is associated with hepatocarcinogenesis $(44,45)$. In HCC cells, higher TGF- $\beta 1$, Smad 7 and NF- $\kappa$ B expression and lower T $\beta$-RII, T $\beta$-RIII and Smad 4 expression have been observed $(46,47)$.

Mechanism of escape from TGF- $\beta$ growth inhibition in HCC cells. Recent studies have mainly focused on the HCC cell mechanism of escape from TGF- $\beta$ growth inhibition since TGF- $\beta$ promotes apoptosis in HCC cells and also activates survival signals, such as AKT (39). The AKT pathway is also 


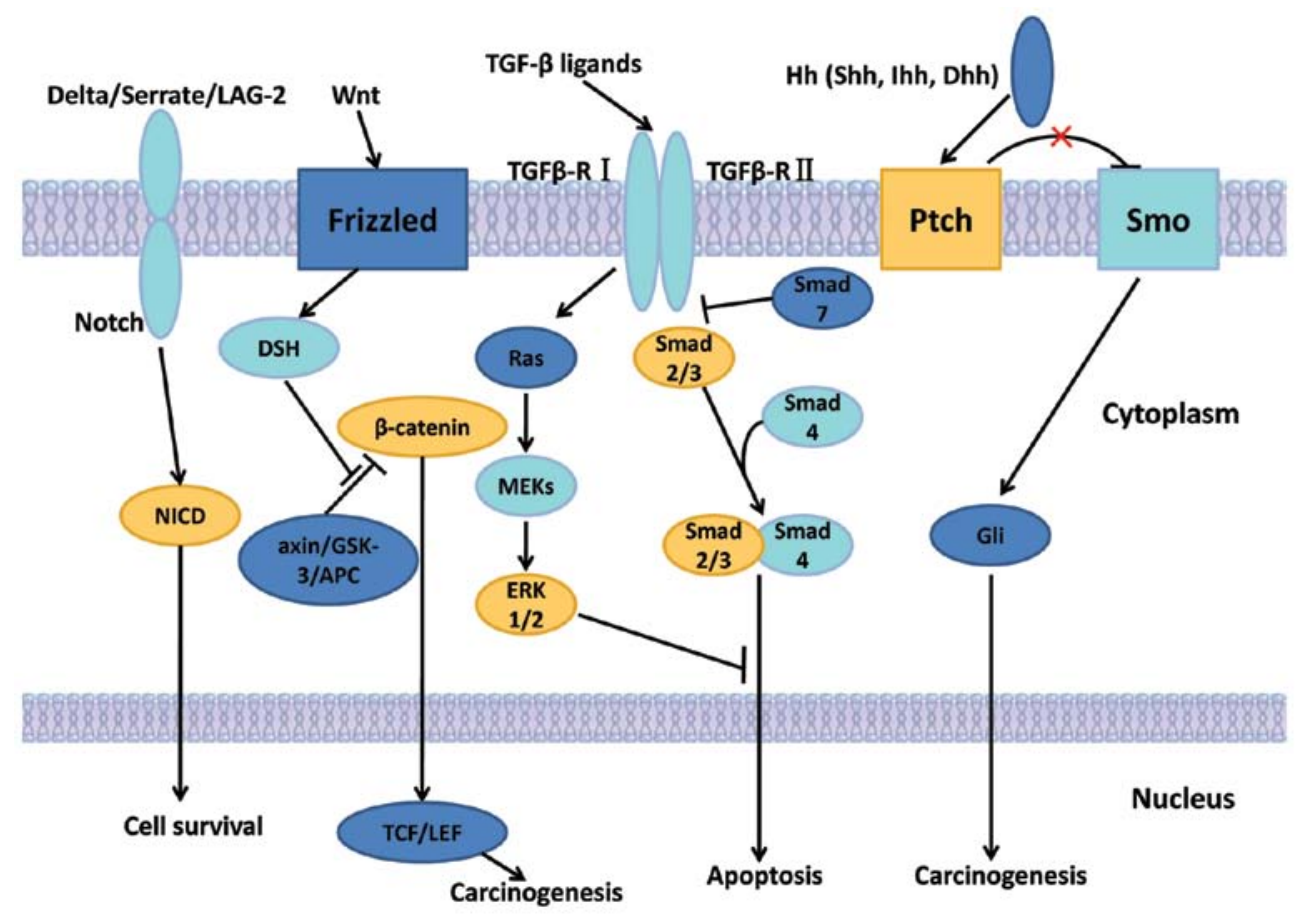

Figure 2. The LCSC signaling pathways. The TGF- $\beta$ signaling pathway, the Wnt signaling pathway, the Notch signaling pathway and the Hedgehog signaling pathway. Abbreviations: Dhh, desert hedgehog; Hh, hedgehog; Ihh, Indian hedgehog; NICD, Notch intracellular domain; Ptch, patched homolog; Shh, sonic the hedgehog homolog; Smo, Smoothened; TGF- $\beta$, transforming growth factor $\beta$.

involved in IL-4-transduced signaling pathways, which are able to protect HCC cells from TGF- $\beta$-induced apoptosis (48). Smad 3 plays a dual role in carcinogenesis, as it promotes apoptosis and is essential for TGF- $\beta$-mediated immune suppression (49). Smad 7, another member of the TGF- $\beta$ signaling pathway, confers resistance to the antiproliferative effects of TGF- $\beta$ on HCC cells by inhibiting the formation of the TGF- $\beta$ induced functional Smad-DNA complex (50). Loss of ELF, an embryonic liver fodrin belonging to the type II $\beta$-spectrin adaptor proteins, is considered an early event in hepatocarcinogenesis and stimulation of angiogenesis in HCC tissue $(51,52)$. TGF- $\beta$-induced apoptosis requires the participation of NADPH oxidase, NOX4, and thus, impairing NOX4 upregulation inhibits TGF- $\beta$-induced cell death in HCC (53). Further exploration of the mechanism showed that NOX4 upregulation was impaired by the overactivation of the MEK/ERK pathway (54). Disabled p53, p21Cip1, or Rb genes may also be involved in the escape from TGF- $\beta$ growth inhibition in HCC cells (55).

TGF- $\beta$ signaling pathway and LCSCs. The cooperation between TGF- $\beta$ and oncogenic RAS activates the nuclear $\beta$-catenin signaling pathway, which causes neoplastic hepatocyte dedifferentiation to immature progenitor cells and facilitates HCC recurrence (56). This evidence not only supports the dedifferentiation theory for the source of LCSCs, but also shows the relationship between TGF- $\beta$ signaling and LCSCs. Activation of IL-6/STAT3, a main signaling pathway in liver stem cells, can induce malignant transformation in liver stem cells along with inactivation of the TGF- $\beta$ signaling pathway $(57,58)$. In addition, downregulation of Socs1 induced activation of STAT3, and this process plays a crucial role in malignant transformation (59).
Wnt signaling pathway. The Wnt signaling pathway plays an important role in embryogenesis and tumor development. It consists of a large number of proteins that interact with each other to regulate the signaling pathway. $\beta$-catenin is a key component in the pathway and is inhibited by a protein complex that includes GSK-3, axin and APC $(60,61)$. Binding of the Wnt proteins to the Frizzled receptors activates the Dishevelled (DSH) protein family (62). Subsequently, DSH inhibits the axin/GSK-3/APC complex, and $\beta$-catenin is able to enter the nucleus to interact with the TCF/LEF family of transcription factors to promote expression of specific genes, such as cyclin D1, Myc and TCF-1 (63-65).

Activation of the canonical Wnt signaling pathway drives tumor formation in liver stem cells $(66,67)$. Recent studies have shown that the expression of $\beta$-catenin was higher in HCC than in non-tumor tissues (68), and inhibition of Wnt-1 signaling caused antitumor effects (69). In addition, the noncanonical Wnt signaling pathway plays an important role in HCC. Yuzugullu et al (70) reported that noncanonical Wnt5a represses noncanonical Wnt signaling. This study suggests that the Wnt pathway is selectively activated or repressed depending on the differentiation stages of HCC cells. Wnt signaling is activated in well-differentiated HCC cells and is repressed in poorly differentiated cell lines. In a subsequent study, Wnt11, a member of the noncanonical cascade, was also able to inhibit HCC cell proliferation and migration (71).

Polycomb-group gene products play a pivotal role in HCC formation and maintenance by modulating the Wnt pathway. Polycomb proteins can form two major complexes: polycomb repressive complex 1 and 2 (PRC1 and PRC2). BMI1, a subunit of PRC1, and EZH2, a subunit of PRC2, are expressed in large quantities within $\mathrm{HCC}$ tissues and enable in vitro $\mathrm{HCC}$ cell 
growth (72). In addition, a definite link between high levels of BMI1 or EZH2 expression and the maintenance of tumor-initiating cells in $\mathrm{HCC}$ has been observed $(73,74)$. Furthermore, the expression of EZH2 activates the Wnt/ $\beta$-catenin signaling pathway by silencing the Wnt antagonists, thereby inducing HCC cell proliferation (75).

The Wnt signaling pathway also plays a crucial role in promoting liver growth and regulating liver stem cells (76,77). Yamashita et al (77) employed a novel prognostic HCC subtype cell line to identify the relationship between Wnt signaling and EpCAM, a hepatic stem cell marker. They concluded that EpCAM was a target gene of Wnt signaling, and that $\operatorname{EpCAM}(+) \mathrm{HCC}$ cells have the ability to both selfrenew and differentiate, which suggests that $\operatorname{EpCAM}(+) \mathrm{HCC}$ cells may be LCSCs (78).

Notch signaling pathway. The Notch signaling pathway involves multiple cell differentiation processes during embryonic development and throughout adulthood. It has also been demonstrated that Notch signaling plays an important role in many types of human cancers, including T-cell leukemia, lymphoma, medulloblastoma and colorectal, pancreatic, mammary, ovarian, lung, gastric, cervical and breast carcinoma (79-82). The involvement of Notch in cancer development is complex, since Notch can function as an oncogene or a tumor suppressor depending on the tissue.

Notch signaling was first highlighted in human T-cell leukemia. Dysregulated Notch signaling can promote tumorigenesis, and direct Notch inhibition has been found to have antiproliferative effects on T-cell acute lymphoblastic leukemia (T-ALL) $(83,84)$. Activated Notch signaling has been observed in a wide variety of breast carcinomas $(85,86)$. High Notch1 protein expression is an early event in breast cancer development and is associated with the HER-2 molecular subtype (87); there is also a general increase in the Notch1, Notch2, Notch4, Jagged1, Jagged 2 and Delta-like 4 protein expression in breast carcinoma (88). Emerging evidence suggests that the Notch signaling pathway may be a potential therapeutic target in breast carcinoma $(89,90)$.

Low expression levels of Notch1/Jagged1 were frequently observed, and downregulation of Notch1/Jagged1 signaling may sustain tumor progression in HCC (68). Upregulation of Notch1 was also shown to retard hepatocarcinogenesis by arresting the cell cycle and inducing apoptosis (91). In addition, high Notch3 and low Notch4 expression levels may be associated with HCC (92).

In some solid tumors, dysregulation of the Notch signaling pathway is correlated with tumor initiation (93-95). These findings suggest that aberrant Notch expression may influence CSC regulation and induce tumorigenesis (96).

Hedgehog signaling pathway. The Hedgehog signaling pathway plays a key role in embryonic development and carcinogenesis. The main members of the Hedgehog signaling pathway include the polypeptide ligands $\mathrm{Hh}$ (Shh, Ihh, Dhh), cell-surface transmembrane receptors (PTCH and SMO) and a downstream transcription factor (Gli). A large number of experiments demonstrate that Hedgehog signaling activation is involved in HCC oncogenesis, proliferation and invasiveness $(97,98)$. Blocking the Hedgehog signaling pathway could inhibit HCC formation by restraining proliferation, inducing apoptosis and repressing c-Myc and cyclin D expression (99).

Members of the Hedgehog signaling pathway perform differently in hepatocarcinogenesis. PTCH (PTCH1), the Hedgehog signaling receptor, is associated with the early stage of HCC formation (100). Smo is considered a prognostic factor for HCC formation and it plays a critical role in hepatocarcinogenesis by mediating c-myc overexpression (101). The basal expression of Gli2, which is regulated by p53, Notch and TGF- $\beta$ signaling, could prime the Hedgehog signaling pathway and lead to HCC tumor formation (102). Activation of the Hedgehog signaling pathway may influence the Wnt signaling pathway by regulating the transcription of a secreted frizzledrelated protein (sFRP-1), which has the ability to suppress Wnt signaling (103). In addition, knockdown of Rab23, an essential negative regulator of the Hedgehog signaling pathway, is reported to suppress HCC cell growth (104).

It has been suggested that Hedgehog signaling pathway activation might be related to LCSCs. In normal liver tissue, the expression of $\mathrm{Hh}$ is low and mature hepatocytes are not Hh-responsive. Omenetti and Diehl (105) found that high levels of Hh were expressed after liver injury and that this favored the survival of Hh-responsive cells, such as myofibroblastic and progenitor cells. During subsequent differentiation, the original Hh-responsive population progeny proliferates and this may lead to hepatic fibrosis and neoplasia. Therefore, the progenitor cells that survived may initiate hepatocarcinogenesis. A recent report also demonstrated that HBV/ $\mathrm{HCV}$ infection induced high $\mathrm{Hh}$ ligand expression levels and Hh-responsive cell proliferation, promoting liver fibrosis and cancer (106). In addition, Hedgehog signaling pathway activation may cause malignant embryonal liver cell transformation in hepatoblastoma (107). In summary, the Hedgehog signaling pathway plays an important role in LCSC regulation.

\section{Markers for liver cancer stem cells}

In order to isolate LCSCs from HCC tissues, several biomarkers have been identified, including CD133, EpCAM and ABCG2. These biomarkers and others are discussed below.

CD133. CD133, which is expressed in hematopoietic and neuronal stem cells, has long been considered an important CSC marker in HCC. In normal liver tissues, CD133(+) cells are related to liver regeneration and may also serve as selfrenewing bipotent primitive hepatic cells (108). Further study showed that CD133(+)CD45(-) cells from chronic liver disease represented a bipotent liver stem cell population at the stage of primary carcinoma formation, which had CSC characteristics (109). Emerging evidence suggests that CD133 expression is a putative marker for LCSCs as follows: i) a small population of CD133(+) cells was observed in HCC tissues (110); ii) $\mathrm{CD} 133(+) \mathrm{HCC}$ cells had a higher proliferative potential and a greater ability to form colonies (111); iii) CD133(+) HCC cells possess the characteristics of progenitor cells (111); iv) the high expression level of 'stemness' genes and the low expression level of the mature hepatocyte markers, glutamine synthetase and cytochrome P450 3A4 were observed in CD133(+) HCC cells as compared with CD133(-) HCC cells $(111,112)$; v) after injection into SCID mice, CD133(+) cells 
from HCC tissue formed tumors, while CD133(-) cells did not (112); vi) CD133 expression may contribute to HCC survival (113); and vii) knockdown of CD133 expression reduces the ability to form colonies and alter the cell cycle distribution in HCC (114). In addition, increased CD133 expression may indicate a poor prognosis and tumor recurrence in patients with HCC (115).

It has been demonstrated that co-expression of CD133 and other cell surface markers could define CSCs. CD133(+) $\mathrm{ALDH}(+)$ cells represent the CSC population in HCC tissue and there is a hierarchical organization in HCC bearing tumorigenic capacity in the following order: CD133(+) ALDH(+) > CD133(+)ALDH(-) > CD133(-)ALDH(-) (116). Higher tumorigenic potential was also observed in CD133(+) CD44(+) HCC cells compared to CD133(+)CD44(-). Therefore, the co-expression of CD133 and CD44 could be considered markers for LCSCs (117).

The relationship between CD133 expression and signaling pathways has been studied extensively. TGF- $\beta 1$ induces CD133 expression in HCC by inhibiting DNMT1 and DNMT3 $\beta$ expression and these CD133(+) cells subsequently initiate tumor formation (118). The Akt/PKB and $\mathrm{Bcl}-2$ pathway is involved in CD133(+)-HCC cell chemoresistance and this pathway could represent a new target for HCC therapy (119).

A differential analysis between the microRNA expression profiles of CD133(+) and CD133(-) liver cancer cells showed a higher miR-130b expression level in CD133(+) cells (120). In addition, miR-130b plays a critical role in maintaining the stem-like characteristics of CD133(+) cancer cells by silencing tumor protein p53-inducible nuclear protein 1 (TP53INP1) (120).

However, the migratory properties do not differ between CD133(+) and CD133(-) HCC cells and the amount of CD133(+) cells is not related to the HCC clinical status (121). Therefore, it is still uncertain whether or not CD133 can serve as a marker for LCSCs.

EрCAM. EpCAM is expressed during early liver development, but not in hepatocytes. EpCAM is also observed in hepatic stem cells and most hepatoblasts (122). Accumulating evidence suggests that EpCAM may be a potential biomarker for LCSCs, and is presented as follows: i) high levels of known hepatic stem cell markers are expressed in $\operatorname{EpCAM}(+)$ cells, whereas mature hepatocyte markers are increased significantly in EpCAM(-) cells (78); ii) compared with EpCAM(-) cells, $\mathrm{EpCAM}(+)$ cells showed a greater colony formation rate (123); iii) $\operatorname{EpCAM}(+)$ cells contain a multipotent cell population, and they can differentiate into both $\operatorname{EpCAM}(+)$ and EpCAM(-) cells (123); iv) after injection into NOD/SCID mice, $\operatorname{EpCAM}(+)$ cells efficiently initiated tumors, while EpCAM(-) cells could not (78); and v) in the HuH7 cell line, EpCAM(+) cells are much more invasive than EpCAM(-) cells (78). Taken together, this information suggests that $\operatorname{EpCAM}(+) \mathrm{HCC}$ cells represent hepatic stem cells and that these cells may also serve as LCSCs.

EpCAM expression is regulated by the Wnt/ $\beta$-catenin signaling pathway. Accumulation of $\beta$-catenin induces EpCAM expression in normal liver tissue and in HCC tissue, while degradation of $\beta$-catenin or inhibition of $\mathrm{Tcf} / \beta$-catenin complex formation suppresses EpCAM expression (77). A novel regulatory relationship between miR-181 and EpCAM(+) HCC cells has been observed; inhibition of miR-181 reduced the amount of $\operatorname{EpCAM}(+)$ cells and their ability to initiate tumors (124). Therefore, miR-181 may serve as a potential therapeutic target for HCC. In addition, EpCAM, the target of $\beta$-catenin and miR-181, contributes to the regulation of several reprogramming genes, including c-MYC, OCT-4, NANOG, SOX 2 and KLF4, thereby playing a critical role in the maintenance of HCC cell 'stemness' $(125,126)$.

ABCG2. Goodell et al (127) first described a type of primitive stem cell, the side population (SP), in the bone marrow; these cells were distinguished by their ability to exclude Hoechst 33342 dye and they defined this characteristic as a side population phenotype. Recently, SP cells have been considered to be CSCs in many types of tumor tissues (128). In HCC, SP cells harbor CSC-like properties, and they may be related to tumorigenesis, metastasis and therapeutic resistance (129-131). In addition, a study of the HCC cell cycle distribution showed that $\mathrm{G}_{0}$ cells were present in the SP fraction and that they may play a crucial role in tumor pathogenesis $(132,133)$.

ABCG2, an ATP binding cassette (ABC) half-transporter that is highly expressed on the SP plasma membrane, efficiently extrudes a wide variety of compounds such as anticancer agents across cell membranes, and is considered to be the determinant of the SP phenotype. Recent evidence suggests that ABCG2 serves as a CSC biomarker in many types of tumors, such as lung cancer, pancreatic cancer and retinoblastoma (133-136). In addition, Zen et al (137) compared ABCG2(+) with ABCG2(-) subpopulations from HCC tissues. The results showed that other progenitor cell markers, such as CK19 and AFP, were mainly located in ABCG2(+) subpopulations and that $\mathrm{ABCG} 2(+)$ cells may play an important role in hepatocarcinogenesis through their ability to generate both ABCG2(+) and ABCG2(-) progenies. Our previous study also supported the potential for ABCG2 to be a LCSC marker (138). Further study explored the mechanism of ABCG2 expression in $\mathrm{HCC}$, demonstrating that the Akt signaling pathways regulated the SP phenotype activity by altering the subcellular localization of ABCG2 and by suppressing Akt signaling that could help overcome ABCG2-induced chemotherapy resistance $(128,139)$.

Other putative markers. CD90, also named Thy-1, is a conserved cell surface protein that can be used as a marker for a variety of stem cells. In precancerous liver tissues, CD90 expression is observed in proliferating bile ductules and its co-expression with CD34 represents hepatic stem cells (140). Yang et al (141) compared CD90(+) cells with CD90(-) cells from HCC cell lines and demonstrated that CD45(-)CD90(+) cells were detected in all of the tumor specimens and in $90 \%$ of the blood samples from HCC patients. These researchers also demonstrated that CD90 expression increased during tumor formation and that CD90(+) cells formed tumor nodules in immunodeficient mice; CD90(+) cells generated tumor nodules after serial transplantation in a second and then in a third group of immunodeficient mice. Therefore, CD90 may be considered to be a marker for LCSCs.

Co-expression of CD90 and CD13 was found to play an important role in hepatocarcinogenesis, and combination of a 
Table I. Putative markers of LCSCs.

\begin{tabular}{|c|c|c|c|c|c|c|}
\hline $\begin{array}{l}\text { Surface } \\
\text { markers }\end{array}$ & $\begin{array}{l}\text { Percentages of cells } \\
\text { expressing markers }^{\mathrm{a}}\end{array}$ & $\begin{array}{l}\text { Minimum no. of cells } \\
\text { for tumor formation }\end{array}$ & $\begin{array}{l}\text { Injection } \\
\text { site of mice }\end{array}$ & Strain & Latency & Ref. \\
\hline CD133(+)ALDH(+) & $0.94-55.71 \%$ & 500 & s.c. & SCID & 82 days & (116) \\
\hline CD133(+) & $0.1-2.0 \%$ & 100 & i.p. & $\mathrm{BNX}$ & 10 weeks & (110) \\
\hline CD133(+) & $0.10-93.18 \%$ & 100 & s.c. & NOD/SCID & 3 months & (117) \\
\hline CD133(+)CD44(+) & $0.09-1.88 \%$ & 100 & s.c. & NOD/SCID & 2 months & (117) \\
\hline $\operatorname{EpCAM}(+)$ & $0.7-99.6 \%$ & 100 & s.c. & NOG & 6-7 weeks & (123) \\
\hline ABCG2 (SP cells) & $0.25-0.80 \%$ & 1000 & s.c. & NOD/SCID & 16 weeks & $(129)$ \\
\hline CD90(+) & $0.04-2.34 \%$ & 500 & s.c. & SCID/Beige & 3 months & (141) \\
\hline CD90(+)CD44(+) & $0.02-2.53 \%$ & 500 & s.c. & SCID/Beige & 3 months & (141) \\
\hline
\end{tabular}

${ }^{a}$ The numbers represent the percentages of cells expressing the markers in different cell lines. The cell lines used are as follows: CD133(+)ALDH(+) (HepG2, Huh7, PLC8024, Hep3B, H2M); CD133(+) in ref. 110 (SMMC-7721); CD133(+) in ref. 117 (Huh7, SMMC-7721, MHCC-LM3, MHCC-97L, HepG2, Hep3B); CD133(+)CD44(+) (Huh7, SMMC-7721, MHCC-LM3, MHCC-97L); EpCAM(+) (Hep3B, Huh7, HepG2, PLC/ PRF/5, Li7); ABCG2 (Huh7, PLC/PRF/5); CD90(+) (HepG2, Hep3B, PLC, Huh7, MHCC97L, MHCC97H); CD90(+)CD44(+) (HepG2, Hep3B, PLC, Huh7, MHCC97L, MHCC97H). LCSCs, liver cancer stem cells; s.c., subcutaneous; i.p., intraperitoneal; SCID, severe combined immunodeficient; BNX, beige/nude/XID; NOD/SCID, non-obese diabetic/severe combined immunodeficient; NOG, NOD/scid/ $\gamma \mathrm{cnull}$.

CD13 inhibitor and a CD90 inhibitor drastically reduced tumor volume compared with either agent alone. In addition, CD13(+) cells demonstrated CSC characteristics such as proliferation, formation of cellular clusters in cancer foci and the ability to survive during treatment (142). Given these results, CD13 is a potential marker for LCSCs.

Cytokeratin 19 (CK19), a member of the keratin family, is a stemness-related marker. CK19 is expressed in normal human liver bile duct cells and is also observed scattered in the parenchyma of cirrhotic livers and within HCCs $(143,144)$. Compared with CK19(-) cells, CK19(+) early lesions and advanced HCCs contain genetic changes consistent with remodeling toward a differentiated phenotype, and they are an important predictive factor for prognosis, patient survival and tumor recurrence (145). The expression of epithelialmesenchymal transition (EMT)-related proteins, which play a pivotal role in the tumor-cell invasion process, is increased in CK19(+) HCC cells; therefore, CK19(+) HCC cells demonstrate high invasive ability (146).

OV6, a hepatic progenitor cell marker, has recently been regarded as a putative marker for LCSCs. OV6(+) HCC cells demonstrate greater chemoresistance and a greater ability to form tumors in vivo compared to OV6(-) cells (147). Activation of the Wnt pathway tends to give rise to an increase in the proportion of OV6(+) cells, and inhibition of $\beta$-catenin signaling suppresses OV6 expression within HCC cells (147); therefore, the OV6 expression is regulated by the Wnt pathway.

In addition, the expression of other markers, such as CD44, DLK1, Oct4, Nanog, c-kit and Ezmin, may also be related to LCSCs (144,148-152). However, the exact pattern of LCSC marker expression is still unknown. Jabari et al (153) demonstrated that different HCC cell lines express different stem cell markers. The classical cholangiocellular type (Huh-7, Huh-7 pcDNA3.1, Hep3B) expressed CK7/19, $\beta$-catenin and CD34; a dedifferentiated mesenchymal-proliferative type (Huh-7 5-15) was characterized by CK19, vimentin and Ki-67; a dedifferentiated embryonic-development type (Hep3B implanted in
Matrigel) expressed CK19, $\beta$-catenin and PTC; and a classical HCC type (HepG2) expressed CK18/19 and $\beta$-catenin. In addition, $\operatorname{EpCAM}(+)$ cells have a greater capacity to initiate tumors than do CD133(+) cells in the Huh1 cell line, while $\operatorname{EpCAM(+)~and~CD133(+)~cells~showed~similar~tumorigenic~}$ ability in the Huh7 cell line (78). Therefore, determination of LCSC markers requires further research. A summary of putative LCSC markers is provided in Table I.

\section{Discussion}

Although the exact mechanism that controls hepatocarcinogenesis remains unclear, the 'cancer stem cell' theory has been proposed as a potential explanation. LCSCs, a rare population in HCC cells with stem-like characteristics, are thought to be responsible for oncogenic cell transformation.

Dysregulation of signaling pathways such as TGF- $\beta$, Wnt, Notch and Hedgehog plays a crucial role in HCC formation and LCSC maintenance. Recent research has shown that additional factors also contribute to this progression, especially microRNAs. In addition to miR-130b and miR-181 mentioned above, other microRNAs, which participate in cancer cell 'stemness', have also been identified. LIN28, a miRNA-binding protein, is expressed without restriction in embryonic stem cells and various human cancer cells. LIN28 was found to be one of the reprogramming factors, which are able to reprogram somatic cells to pluripotent stem cells (154). Under physiological conditions, the miRNAs let-7, mir-125, mir-9 and mir-30 negatively regulate LIN28 expression and the downregulation of these miRNAs may lead to LIN28 overexpression in tumors (155). In addition, high expression levels of LIN28 can promote tumor formation and malignant transformation by repressing the let-7 family miRNA expression (156). Let-7 is sufficient to negatively regulate LIN28 via a feedback loop (157). In tumor tissue, LIN28 upregulation is tightly linked to a high proportion of ALDH(+) cells, which are regarded as CSC representatives, and LIN28 plays a crucial role in the maintenance of ALDH(+) 
cancer cells. Changes in the LIN28/let-7 regulatory loop induce the 'reprogramming-like' process in tumors, which may explain the formation of CSCs (157).

Numerous markers have been used to identify LCSCs, and these markers may be putative therapeutic targets in HCC. Immunotherapy may also be an effective way to treat $\mathrm{HCC}$ by targeting these biomarkers. CD133(+) HCC cells have long been regarded as potential LCSCs and an anti-CD133 antibody conjugated to a cytotoxic drug is reported to inhibit HCC cell proliferation in vitro (158); therefore, CD133(+) cancer cells may be considered a novel target for HCC therapy. An antiCD44 antibody-mediated liposomal nanoparticle, containing a triple fusion gene (herpes simplex virus truncated thymidine kinase, renilla luciferase and red fluorescent protein), can be used in gene therapy and in the molecular imaging of HCC (159). CD13(+) HCC cells are able to resist regular ROS-inducing chemoradiation therapy as CD13 protects HCC cells from ROS-induced DNA damage. Therefore, a combination of a CD13 inhibitor and ROS-inducing chemoradiation therapy may enhance treatment effectiveness (142). A large amount of circulating CSCs, represented by CD45(+)CD90(+) CD44(+) HCC cells, is tightly linked to an increased possibility of HCC recurrence after hepatectomy, which means these CSCs may be a potential target for the prevention of HCC recurrence (160).

\section{Acknowledgements}

This study was supported by the Jiangsu Science Foundation of China (no. LW201008) and the Nanjing Science Foundation of China (no. ZKX08025).

\section{References}

1. Yu MC and Yuan JM: Environmental factors and risk for hepatocellular carcinoma. Gastroenterology 127 (Suppl 1): S72-S78, 2004.

2. Bosch FX, Ribes J, Cleries R and Diaz M: Epidemiology of hepatocellular carcinoma. Clin Liver Dis 9: 191-211, 2005.

3. Gomaa AI, Khan SA, Toled ano MB, Waked I and TaylorRobinson SD: Hepatocellular carcinoma: epidemiology, risk factors and pathogenesis. World J Gastroenterol 14: 4300-4308, 2008.

4. Shariff MI, Cox IJ, Gomaa AI, Khan SA, Gedroyc W and TaylorRobinson SD: Hepatocellular carcinoma: current trends in worldwide epidemiology, risk factors, diagnosis and therapeutics. Expert Rev Gastroenterol Hepatol 3: 353-367, 2009.

5. Kumar M, Kumar R, Hissar SS, et al: Risk factors analysis for hepatocellular carcinoma in patients with and without cirrhosis: a case-control study of 213 hepatocellular carcinoma patients from India. J Gastroenterol Hepatol 22: 1104-1111, 2007.

6. Carr BI: Hepatocellular carcinoma: current management and future trends. Gastroenterology 127 (Suppl 1): S218-S224, 2004.

7. Kassahun WT, Fangmann J, Harms J, Hauss J and Bartels M: Liver resection and transplantation in the management of hepatocellular carcinoma: a review. Exp Clin Transplant 4: 549-558, 2006.

8. Witjes CD, Verhoef C, Verheul HM and Eskens FA: Systemic treatment in hepatocellular carcinoma; 'A small step for man...' Neth J Med 67: 86-90, 2009.

9. Reya T, Morrison SJ, Clarke MF and Weissman IL: Stem cells, cancer, and cancer stem cells. Nature 414: 105-111, 2001.

10. Bonnet D and Dick JE: Human acute myeloid leukemia is organized as a hierarchy that originates from a primitive hematopoietic cell. Nat Med 3: 730-737, 1997.

11. Al-Hajj M, Wicha MS, Benito-Hernandez A, Morrison SJ and Clarke MF: Prospective identification of tumorigenic breast cancer cells. Proc Natl Acad Sci USA 100: 3983-3988, 2003.
12. Collins AT, Berry PA, Hyde C, Stower MJ and Maitland NJ: Prospective identification of tumorigenic prostate cancer stem cells. Cancer Res 65: 10946-10951, 2005.

13. Klarmann GJ, Hurt EM, Mathews LA, et al: Invasive prostate cancer cells are tumor initiating cells that have a stem cell-like genomic signature. Clin Exp Metastasis 26: 433-446, 2009.

14. Kim CF, Jackson EL, Woolfenden AE, et al: Identification of bronchioalveolar stem cells in normal lung and lung cancer. Cell 121: 823-835, 2005.

15. Eramo A, Lotti F, Sette G, et al: Identification and expansion of the tumorigenic lung cancer stem cell population. Cell Death Differ 15: 504-514, 2008.

16. O'Brien CA, Pollett A, Gallinger S and Dick JE: A human colon cancer cell capable of initiating tumour growth in immunodeficient mice. Nature 445: 106-110, 2007.

17. Chu P, Clanton DJ, Snipas TS, et al: Characterization of a subpopulation of colon cancer cells with stem cell-like properties. Int J Cancer 124: 1312-1321, 2009.

18. Li C, Heidt DG, Dalerba P, et al: Identification of pancreatic cancer stem cells. Cancer Res 67: 1030-1037, 2007.

19. Li C, Lee CJ and Simeone DM: Identification of human pancreatic cancer stem cells. Methods Mol Biol 568: 161-173, 2009.

20. Singh SK, Clarke ID, Terasaki M, et al: Identification of a cancer stem cell in human brain tumors. Cancer Res 63: 5821-5828, 2003.

21. Rahman R, Heath R and Grundy R: Cellular immortality in brain tumours: an integration of the cancer stem cell paradigm. Biochim Biophys Acta 1792: 280-288, 2009.

22. Sun YL, Yin SY, Xie HY, et al: Stem-like cells in hepatitis B virus-associated cirrhotic livers and adjacent tissue to hepatocellular carcinomas possess the capacity of tumorigenicity. J Gastroenterol Hepatol 23: 1280-1286, 2008.

23. Roskams TA, Theise ND, Balabaud C, et al: Nomenclature of the finer branches of the biliary tree: canals, ductules, and ductular reactions in human livers. Hepatology 39: 1739-1745, 2004.

24. Petersen BE, Bowen WC, Patrene KD, et al: Bone marrow as a potential source of hepatic oval cells. Science 284: 1168-1170, 1999.

25. Shi XL, Gu JY, Han B, Xu HY, Fang L and Ding YT: Magnetically labeled mesenchymal stem cells after autologous transplantation into acutely injured liver. World J Gastroenterol 16: 3674-3679, 2010.

26. Theise ND, Nimmakayalu M, Gardner R, et al: Liver from bone marrow in humans. Hepatology 32: 11-16, 2000.

27. Rowe PM: Chronic Lyme disease: the debate goes on. Lancet 355: 1436, 2000.

28. Bae SH, Choi JY, Yoon SK, et al: Thy1-positive bone marrow stem cells express liver-specific genes in vitro and can mature into hepatocytes in vivo. Hepatol Int 2: 63-71, 2008.

29. Gournay J, Auvigne I, Pichard V, Ligeza C, Bralet MP and Ferry N: In vivo cell lineage analysis during chemical hepatocarcinogenesis in rats using retroviral-mediated gene transfer: evidence for dedifferentiation of mature hepatocytes. Lab Invest 82: 781-788, 2002.

30. Bralet MP, Pichard V and Ferry N: Demonstration of direct lineage between hepatocytes and hepatocellular carcinoma in diethylnitrosamine-treated rats. Hepatology 36: 623-630, 2002.

31. Dumble ML, Croager EJ, Yeoh GC and Quail EA: Generation and characterization of p53 null transformed hepatic progenitor cells: oval cells give rise to hepatocellular carcinoma. Carcinogenesis 23: 435-445, 2002.

32. Fujii T, Zen Y, Harada K, et al: Participation of liver cancer stem/ progenitor cells in tumorigenesis of scirrhous hepatocellular carcinoma - human and cell culture study. Hum Pathol 39: 1185$1196,2008$.

33. Nomoto $\mathrm{K}$, Tsuneyama $\mathrm{K}$, Cheng $\mathrm{C}$, et al: Intrahepatic cholangiocarcinoma arising in cirrhotic liver frequently expressed p63-positive basal/stem-cell phenotype. Pathol Res Pract 202: 71-76, 2006.

34. Tanaka S, Yamamoto T, Tanaka H, et al: Potentiality of combined hepatocellular and intrahepatic cholangiocellular carcinoma originating from a hepatic precursor cell: immunohistochemical evidence. Hepatol Res 32: 52-57, 2005.

35. Zhang F, Chen XP, Zhang W, et al: Combined hepatocellular cholangiocarcinoma originating from hepatic progenitor cells: immunohistochemical and double-fluorescence immunostaining evidence. Histopathology 52: 224-232, 2008.

36. Komuta M, Spee B, Vander Borght S, et al: Clinicopathological study on cholangiolocellular carcinoma suggesting hepatic progenitor cell origin. Hepatology 47: 1544-1556, 2008. 
37. de Lima VM, Oliveira CP, Alves VA, et al: A rodent model of NASH with cirrhosis, oval cell proliferation and hepatocellular carcinoma. J Hepatol 49: 1055-1061, 2008.

38. Grozdanov PN, Yovchev MI and Dabeva MD: The oncofetal protein glypican-3 is a novel marker of hepatic progenitor/oval cells. Lab Invest 86: 1272-1284, 2006.

39. Caja L, Ortiz C, Bertran E, et al: Differential intracellular signalling induced by TGF-beta in rat adult hepatocytes and hepatoma cells: implications in liver carcinogenesis. Cell Signal 19: 683-694, 2007.

40. Herzer K, Grosse-Wilde A, Krammer PH, Galle PR and Kanzler S: Transforming growth factor-beta-mediated tumor necrosis factor-related apoptosis-inducing ligand expression and apoptosis in hepatoma cells requires functional cooperation between Smad proteins and activator protein-1. Mol Cancer Res 6: 1169-1177, 2008.

41. Wang CL, Wan YL, Liu YC and Huang ZQ: TGF-beta1/ SMAD signaling pathway mediates p53-dependent apoptosis in hepatoma cell lines. Chin Med Sci J 21: 33-35, 2006.

42. Yang YA, Zhang GM, Feigenbaum L and Zhang YE: Smad3 reduces susceptibility to hepatocarcinoma by sensitizing hepatocytes to apoptosis through downregulation of Bcl-2. Cancer Cell 9: 445-457, 2006

43. Kiyono K, Suzuki HI, Matsuyama H, et al: Autophagy is activated by TGF-beta and potentiates TGF-beta-mediated growth inhibition in human hepatocellular carcinoma cells. Cancer Res 69 8844-8852, 2009.

44. Mazzocca A,Fransvea E,Lavezzari G, Antonaci S and Giannelli G: Inhibition of transforming growth factor beta receptor I kinase blocks hepatocellular carcinoma growth through neo-angiogenesis regulation. Hepatology 50: 1140-1151, 2009.

45. Mikula M, Proell V, Fischer AN and Mikulits W: Activated hepatic stellate cells induce tumor progression of neoplastic hepatocytes in a TGF-beta dependent fashion. J Cell Physiol 209: $560-567,2006$

46. Bae HJ, Eun JW, Noh JH, et al: Down-regulation of transforming growth factor beta receptor type III in hepatocellular carcinoma is not directly associated with genetic alterations or loss of heterozygosity. Oncol Rep 22: 475-480, 2009.

47. Ji GZ, Wang XH, Miao L, et al: Role of transforming growth factor-beta1-smad signal transduction pathway in patients with hepatocellular carcinoma. World J Gastroenterol 12: 644-648, 2006.

48. Lin SJ, Chang C, Ng AK, Wang SH, Li JJ and Hu CP: Prevention of TGF-beta-induced apoptosis by interleukin- 4 through Akt activation and p70S6K survival signaling pathways. Apoptosis 12: 1659-1670, 2007.

49. Millet $C$ and Zhang YE: Roles of Smad3 in TGF-beta signaling during carcinogenesis. Crit Rev Eukaryot Gene Expr 17: 281-293, 2007.

50. Zhang S, Fei T, Zhang L, et al: Smad7 antagonizes transforming growth factor beta signaling in the nucleus by interfering with functional Smad-DNA complex formation. Mol Cell Biol 27: 4488-4499, 2007.

51. Kitisin K, Ganesan N, Tang Y, et al: Disruption of transforming growth factor-beta signaling through beta-spectrin ELF leads to hepatocellular cancer through cyclin D1 activation. Oncogene 26: 7103-7110, 2007

52. Baek HJ, Lim SC, Kitisin K, et al: Hepatocellular cancer arises from loss of transforming growth factor beta signaling adaptor protein embryonic liver fodrin through abnormal angiogenesis. Hepatology 48: 1128-1137, 2008.

53. Carmona-Cuenca I, Roncero C, Sancho P, et al: Upregulation of the NADPH oxidase NOX4 by TGF-beta in hepatocytes is required for its pro-apoptotic activity. J Hepatol 49: 965-976, 2008.

54. Caja L, Sancho P, Bertran E, Iglesias-Serret D, Gil J and Fabregat I: Overactivation of the MEK/ERK pathway in liver tumor cells confers resistance to TGF-\{beta\}-induced cell death through impairing up-regulation of the NADPH oxidase NOX4 Cancer Res 69: 7595-7602, 2009.

55. Sheahan S, Bellamy CO, Dunbar DR, Harrison DJ and Prost $S$ Deficiency of G1 regulators P53, P21Cip1 and/or pRb decreases hepatocyte sensitivity to TGFbeta cell cycle arrest. BMC Cancer 7: 215, 2007.

56. Zulehner G, Mikula M, Schneller D, et al: Nuclear beta-catenin induces an early liver progenitor phenotype in hepatocellular carcinoma and promotes tumor recurrence. Am J Pathol 176: 472-481, 2010

57. Tang Y, Kitisin K, Jogunoori W, et al: Progenitor/stem cells give rise to liver cancer due to aberrant TGF-beta and IL-6 signaling. Proc Natl Acad Sci USA 105: 2445-2450, 2008.
58. Lin L, Amin R, Gallicano GI, et al: The STAT3 inhibitor NSC 74859 is effective in hepatocellular cancers with disrupted TGF-beta signaling. Oncogene 28: 961-972, 2009.

59. Bagnyukova TV, Tryndyak VP, Muskhelishvili L, Ross SA, Beland FA and Pogribny IP: Epigenetic downregulation of the suppressor of cytokine signaling 1 (Socs1) gene is associated with the STAT3 activation and development of hepatocellular carcinoma induced by methyl-deficiency in rats. Cell Cycle 7: 3202-3210, 2008

60. Dajani R, Fraser E, Roe SM, et al: Structural basis for recruitment of glycogen synthase kinase 3beta to the axin-APC scaffold complex. EMBO J 22: 494-501, 2003.

61. Ha NC, Tonozuka T, Stamos JL, Choi HJ and Weis WI: Mechanism of phosphorylation-dependent binding of APC to beta-catenin and its role in beta-catenin degradation. Mol Cell 15: 511-521, 2004.

62. Tauriello DV, Jordens I, Kirchner K, et al: Wnt/ $\beta$-catenin signaling requires interaction of the Dishevelled DEP domain and $\mathrm{C}$ terminus with a discontinuous motif in Frizzled. Proc Natl Acad Sci USA 109: E812-E820,2012.

63. Tetsu $\mathrm{O}$ and McCormick F: Beta-catenin regulates expression of cyclin D1 in colon carcinoma cells. Nature 398: 422-426, 1999.

64. Yochum GS, Sherrick CM, Macpartlin M and Goodman RH A beta-catenin/TCF-coordinated chromatin loop at MYC integrates 5' and 3' Wnt responsive enhancers. Proc Natl Acad Sci USA 107: 145-150, 2010.

65. Staal FJ, Meeldijk J, Moerer P, et al: Wnt signaling is required for thymocyte development and activates Tcf-1 mediated transcription. Eur J Immunol 31: 285-293, 2001.

66. Taniguchi $\mathrm{H}$ and Chiba T: Stem cells and cancer in the liver. Dis Markers 24: 223-229, 2008

67. Takigawa $\mathrm{Y}$ and Brown AM: Wnt signaling in liver cancer. Curr Drug Targets 9: 1013-1024, 2008.

68. Wang M, Xue L, Cao Q, et al: Expression of Notch1, Jagged1 and beta-catenin and their clinicopathological significance in hepatocellular carcinoma. Neoplasma 56: 533-541, 2009.

69. Wei W, Chua MS, Grepper S and So SK: Blockade of Wnt-1 signaling leads to anti-tumor effects in hepatocellular carcinoma cells. Mol Cancer 8: 76, 2009.

70. Yuzugullu H, Benhaj K, Ozturk N, et al: Canonical Wnt signaling is antagonized by noncanonical Wnt5a in hepatocellular carcinoma cells. Mol Cancer 8: 90, 2009.

71. Toyama T, Lee HC, Koga H, Wands JR and Kim M: Noncanonical Wnt11 inhibits hepatocellular carcinoma cell proliferation and migration. Mol Cancer Res 8: 254-265, 2010.

72. Yonemitsu Y, Imazeki F, Chiba T, et al: Distinct expression of polycomb group proteins EZH2 and BMI1 in hepatocellular carcinoma. Hum Pathol 40: 1304-1311, 2009.

73. Chiba T, Miyagi S, Saraya A, et al: The polycomb gene product BMI1 contributes to the maintenance of tumor-initiating side population cells in hepatocellular carcinoma. Cancer Res 68: 7742-7749, 2008

74. Chiba T, Suzuki E, Negishi M, et al: 3-Deazaneplanocin A is a promising therapeutic agent for the eradication of tumor-initiating hepatocellular carcinoma cells. Int J Cancer 130: 2557-2567, 2012.

75. Cheng AS, Lau SS, Chen Y, et al: EZH2-mediated concordant repression of Wnt antagonists promotes $\beta$-catenin-dependent hepatocarcinogenesis. Cancer Res 71: 4028-4039, 2011.

76. Zaret KS: Genetic programming of liver and pancreas progenitors: lessons for stem-cell differentiation. Nat Rev Genet 9: 329-340, 2008.

77. Yamashita T, Budhu A, Forgues M and Wang XW: Activation of hepatic stem cell marker EpCAM by Wnt-beta-catenin signaling in hepatocellular carcinoma. Cancer Res 67: 10831-10839, 2007.

78. Yamashita T, Ji J, Budhu A, et al: EpCAM-positive hepatocellular carcinoma cells are tumor-initiating cells with stem/progenitor cell features. Gastroenterology 136: 1012-1024, 2009.

79. Yin L, Velazquez OC and Liu ZJ: Notch signaling: emerging molecular targets for cancer therapy. Biochem Pharmacol 80: 690-701, 2010.

80. Sharma VM, Draheim KM and Kelliher MA: The Notch1/c-Myc pathway in T cell leukemia. Cell Cycle 6: 927-930, 2007.

81. Moserle L, Ghisi M, Amadori A and Indraccolo S: Side population and cancer stem cells: therapeutic implications. Cancer Lett 288: 1-9, 2010.

82. Wu WK, Cho CH, Lee CW, et al: Dysregulation of cellular signaling in gastric cancer. Cancer Lett 295: 144-153, 2010.

83. Moellering RE, Cornejo M, Davis TN, et al: Direct inhibition of the NOTCH transcription factor complex. Nature 462: 182-188, 2009. 
84. Arora PS and Ansari AZ: Chemical biology: A Notch above other inhibitors. Nature 462: 171-173, 2009.

85. Farnie G and Clarke RB: Mammary stem cells and breast cancer - role of Notch signalling. Stem Cell Rev 3: 169-175, 2007.

86. Stylianou S, Clarke RB and Brennan K: Aberrant activation of notch signaling in human breast cancer. Cancer Res 66: 1517-1525, 2006.

87.Zardawi SJ, Zardawi I, McNeil CM, et al: High Notch1 protein expression is an early event in breast cancer development and is associated with the HER-2 molecular subtype. Histopathology 56: 286-296, 2010.

88. Mittal S, Subramanyam D, Dey D, Kumar RV and Rangarajan A: Cooperation of Notch and Ras/MAPK signaling pathways in human breast carcinogenesis. Mol Cancer 8: 128, 2009.

89. Hirose $\mathrm{H}$, Ishii $\mathrm{H}$, Mimori $\mathrm{K}$, et al: Notch pathway as candidate therapeutic target in Her2/Neu/ErbB2 receptor-negative breast tumors. Oncol Rep 23: 35-43, 2010.

90. Korkaya $\mathrm{H}$ and Wicha MS: HER-2, notch, and breast cancer stem cells: targeting an axis of evil. Clin Cancer Res 15: 1845-1847, 2009.

91. Wang C, Qi R, Li N, et al: Notch1 signaling sensitizes tumor necrosis factor-related apoptosis-inducing ligand-induced apoptosis in human hepatocellular carcinoma cells by inhibiting Akt/Hdm2-mediated p53 degradation and up-regulating p53-dependent DR5 expression. J Biol Chem 284: 16183-16190, 2009.

92. Gramantieri L, Giovannini C, Lanzi A, et al: Aberrant Notch3 and Notch4 expression in human hepatocellular carcinoma. Liver Int 27: 997-1007, 2007.

93. Sikandar SS, Pate KT, Anderson S, et al: NOTCH signaling is required for formation and self-renewal of tumor-initiating cells and for repression of secretory cell differentiation in colon cancer. Cancer Res 70: 1469-1478, 2010.

94. Harrison H, Farnie G, Howell SJ, et al: Regulation of breast cancer stem cell activity by signaling through the Notch 4 receptor. Cancer Res 70: 709-718, 2010.

95.Zhen Y, Zhao S, Li Q, Li Y and Kawamoto K: Arsenic trioxidemediated Notch pathway inhibition depletes the cancer stem-like cell population in gliomas. Cancer Lett 292: 64-72, 2010.

96. Hambardzumyan D, Becher OJ and Holland EC: Cancer stem cells and survival pathways. Cell Cycle 7: 1371-1378, 2008.

97. Huang S, He J, Zhang X, et al: Activation of the hedgehog pathway in human hepatocellular carcinomas. Carcinogenesis 27: 1334-1340, 2006

98. Cheng WT, Xu K, Tian DY, Zhang ZG, Liu LJ and Chen Y: Role of Hedgehog signaling pathway in proliferation and invasiveness of hepatocellular carcinoma cells. Int J Oncol 34: 829-836, 2009

99.Patil MA, Zhang J, Ho C, Cheung ST, Fan ST and Chen X: Hedgehog signaling in human hepatocellular carcinoma. Cancer Biol Ther 5: 111-117, 2006

100.Fu X, Wang Q, Chen X, et al: Expression patterns and polymorphisms of PTCH in Chinese hepatocellular carcinoma patients. Exp Mol Pathol 84: 195-199, 2008.

101. Sicklick JK, Li YX, Jayaraman A, et al: Dysregulation of the Hedgehog pathway in human hepatocarcinogenesis. Carcinogenesis 27: 748-757, 2006.

102. Katoh $\mathrm{Y}$ and Katoh $\mathrm{M}$ : Integrative genomic analyses on GLI2: mechanism of Hedgehog priming through basal GLI2 expression, and interaction map of stem cell signaling network with P53. Int J Oncol 33: 881-886, 2008 .

103.He J, Sheng T, Stelter AA, et al: Suppressing Wnt signaling by the hedgehog pathway through sFRP-1. J Biol Chem 281 : 35598-35602, 2006.

104. Liu YJ, Wang Q, Li W, et al: Rab23 is a potential biological target for treating hepatocellular carcinoma. World J Gastroenterol 13 1010-1017, 2007.

105. Omenetti A and Diehl AM: The adventures of sonic hedgehog in development and repair. II. Sonic hedgehog and liver development, inflammation, and cancer. Am J Physiol Gastrointes Liver Physiol 294: G595-G598, 2008.

106. Pereira Tde A, Witek RP, Syn WK, et al: Viral factors induce Hedgehog pathway activation in humans with viral hepatitis, cirrhosis, and hepatocellular carcinoma. Lab Invest 90: 1690-1703, 2010.

107. Eichenmuller M, Gruner I, Hagl B, et al: Blocking the hedgehog pathway inhibits hepatoblastoma growth. Hepatology 49 : 482-490, 2009.

108. Suzuki A, Sekiya S, Onishi M, et al: Flow cytometric isolation and clonal identification of self-renewing bipotent hepatic progenitor cells in adult mouse liver. Hepatology 48: 1964-1978, 2008.
109. Rountree CB, Ding W, He L and Stiles B: Expansion of CD133expressing liver cancer stem cells in liver-specific phosphatase and tensin homolog deleted on chromosome 10-deleted mice. Stem Cells 27: 290-299, 2009.

110. Yin S, Li J, Hu C, et al: CD133 positive hepatocellular carcinoma cells possess high capacity for tumorigenicity. Int J Cancer 120: 1444-1450, 2007.

111. Ma S, Chan KW, Hu L, et al: Identification and characterization of tumorigenic liver cancer stem/progenitor cells Gastroenterology 132: 2542-2556, 2007.

112. Suetsugu A, Nagaki M, Aoki H, Motohashi T, Kunisada T and Moriwaki H: Characterization of $\mathrm{CD}_{133^{+}}$hepatocellular carcinoma cells as cancer stem/progenitor cells. Biochem Biophys Res Commun 351: 820-824, 2006.

113. Kohga K, Tatsumi T, Takehara T, et al: Expression of CD133 confers malignant potential by regulating metalloproteinases in human hepatocellular carcinoma. J Hepatol 52: 872-879, 2010.

114. Yao J, Zhang T, Ren J, Yu M and Wu G: Effect of CD133/ prominin-1 antisense oligodeoxynucleotide on in vitro growth characteristics of Huh-7 human hepatocarcinoma cells and U251 human glioma cells. Oncol Rep 22: 781-787, 2009.

115. Song W, Li H, Tao K, et al: Expression and clinical significance of the stem cell marker CD133 in hepatocellular carcinoma. Int J Clin Pract 62: 1212-1218, 2008.

116. Ma S, Chan KW, Lee TK, et al: Aldehyde dehydrogenase discriminates the CD133 liver cancer stem cell populations. Mol Cancer Res 6: 1146-1153, 2008.

117. Zhu Z, Hao X, Yan M, et al: Cancer stem/progenitor cells are highly enriched in $\mathrm{CD} 133^{+} \mathrm{CD} 44^{+}$population in hepatocellular carcinoma. Int J Cancer 126: 2067-2078, 2010

118. You H, Ding W and Rountree CB: Epigenetic regulation of cancer stem cell marker CD133 by transforming growth factorbeta. Hepatology 51: 1635-1644, 2010.

119. Ma S, Lee TK, Zheng BJ, Chan KW and Guan XY: CD133+ HCC cancer stem cells confer chemoresistance by preferential expression of the Akt/PKB survival pathway. Oncogene 27: 1749-1758, 2008.

120. Ma S, Tang KH, Chan YP, et al: miR-130b promotes CD133(+) liver tumor-initiating cell growth and self-renewal via tumor protein 53-induced nuclear protein 1. Cell Stem Cell 7: 694-707, 2010.

121. Salnikov AV, Kusumawidjaja G, Rausch V, et al: Cancer stem cell marker expression in hepatocellular carcinoma and liver metastases is not sufficient as single prognostic parameter. Cancer Lett 275: 185-193, 2009.

122. Schmelzer E and Reid LM: EpCAM expression in normal, nonpathological tissues. Front Biosci 13: 3096-3100, 2008

123. Kimura O, Takahashi T, Ishii N, et al: Characterization of the epithelial cell adhesion molecule (EpCAM) ${ }^{+}$cell population in hepatocellular carcinoma cell lines. Cancer Sci 101: 2145-2155, 2010.

124. Ji J, Yamashita T, Budhu A, et al: Identification of microRNA-181 by genome-wide screening as a critical player in EpCAM-positive hepatic cancer stem cells. Hepatology 50: 472-480, 2009.

125. Arzumanyan A, Friedman T, Ng IO, Clayton MM, Lian Z and Feitelson MA: Does the hepatitis B antigen HBx promote the appearance of liver cancer stem cells? Cancer Res 71: 3701-3708, 2011.

126. Lu TY, Lu RM, Liao MY, et al: Epithelial cell adhesion molecule regulation is associated with the maintenance of the undifferentiated phenotype of human embryonic stem cells. J Biol Chem 285: 8719-8732, 2010 .

127. Goodell MA, Brose K, Paradis G, Conner AS and Mulligan RC: Isolation and functional properties of murine hematopoietic stem cells that are replicating in vivo. J Exp Med 183: 1797-1806, 1996

128. Ding XW, Wu JH and Jiang CP: ABCG2: a potential marker of stem cells and novel target in stem cell and cancer therapy. Life Sci 86: 631-637, 2010

129. Chiba T, Kita K, Zheng YW, et al: Side population purified from hepatocellular carcinoma cells harbors cancer stem cell-like properties. Hepatology 44: 240-251, 2006

130. Shi GM, Xu Y, Fan J, et al: Identification of side population cells in human hepatocellular carcinoma cell lines with stepwise metastatic potentials. J Cancer Res Clin Oncol 134: 1155-1163, 2008.

131. Zhang N, Li R, Tao KS, et al: Characterization of a stem-like population in hepatocellular carcinoma MHCC97 cells. Oncol Rep 23: 827-831, 2010.

132. Kamohara Y, Haraguchi N, Mimori K, et al: The search for cancer stem cells in hepatocellular carcinoma. Surgery 144: $119-124,2008$ 
133. Qiang GH, Yu DC and Jiang CP: Side population cells and liver cancer stem cells. World Chin J Digestol 18: 971-974, 2010 (In Chinese).

134. Polgar O, Robey RW and Bates SE: ABCG2: structure, function and role in drug response. Expert Opin Drug Metab Toxicol 4: $1-15,2008$.

135. Sarkadi B, Ozvegy-Laczka C, Nemet K and Varadi A: ABCG2 - a transporter for all seasons. FEBS Lett 567: 116-120, 2004.

136. Han B and Zhang JT: Multidrug resistance in cancer chemotherapy and xenobiotic protection mediated by the half ATP-binding cassette transporter ABCG2. Curr Med Chem Anticancer Agents 4: 31-42, 2004.

137.Zen Y, Fujii T, Yoshikawa S, et al: Histological and culture studies with respect to ABCG2 expression support the existence of a cancer cell hierarchy in human hepatocellular carcinoma. Am J Pathol 170: 1750-1762, 2007.

138.Xi Z, Jiang CP and Ding YT: Expression of stem cell marker ABCG2 and its significance in hepatocellular carcinoma tissue and cell lines. World Chin J Digestol 17: 247-252, 2009.

139. Hu C, Li H, Li J, et al: Analysis of ABCG2 expression and side population identifies intrinsic drug efflux in the HCC cell line MHCC-97L and its modulation by Akt signaling. Carcinogenesis 29: 2289-2297, 2008

140. Yang ZF, Ngai P, Ho DW, et al: Identification of local and circulating cancer stem cells in human liver cancer. Hepatology 47: 919-928, 2008.

141. Yang ZF, Ho DW, Ng MN, et al: Significance of CD90 $0^{+}$cancer stem cells in human liver cancer. Cancer Cell 13: 153-166, 2008.

142. Haraguchi $\mathrm{N}$, Ishii $\mathrm{H}$, Mimori K, et al: CD13 is a therapeutic target in human liver cancer stem cells. J Clin Invest 120: 3326-3339, 2010.

143. Oliva J, French BA, Qing X and French SW: The identification of stem cells in human liver diseases and hepatocellular carcinoma. Exp Mol Pathol 88: 331-340, 2010.

144. Martinez-Chantar ML, Lu SC, Mato JM, et al: The role of stem cells/progenitor cells in liver carcinogenesis in glycine $\mathrm{N}$-methyltransferase deficient mice. Exp Mol Pathol 88: 234-237, 2010.

145. Andersen JB, Loi R, Perra A, et al: Progenitor-derived hepatocellular carcinoma model in the rat. Hepatology 51: 1401-1409, 2010.

146. Kim H, Choi GH, Na DC, et al: Human hepatocellular carcinomas with 'stemness'-related marker expression: keratin 19 expression and a poor prognosis. Hepatology 54: 1707-1717, 2011.

147. Yang W, Yan HX, Chen L, et al: Wnt/beta-catenin signaling contributes to activation of normal and tumorigenic liver progenitor cells. Cancer Res 68: 4287-4295, 2008.
148. Xie Z, Choong PF, Poon LF, et al: Inhibition of CD44 expression in hepatocellular carcinoma cells enhances apoptosis, chemosensitivity, and reduces tumorigenesis and invasion. Cancer Chemother Pharmacol 62: 949-957, 2008.

149. Yu F, Hao X, Zhao H, et al: Delta-like 1 contributes to cell growth by increasing the interferon-inducible protein 16 expression in hepatocellular carcinoma. Liver Int 30: 703-714, 2010.

150. Machida K, Tsukamoto H, Mkrtchyan H, et al: Toll-like receptor 4 mediates synergism between alcohol and HCV in hepatic oncogenesis involving stem cell marker Nanog. Proc Natl Acad Sci USA 106: 1548-1553, 2009.

151. Knight B, Tirnitz-Parker JE and Olynyk JK: C-kit inhibition by imatinib mesylate attenuates progenitor cell expansion and inhibits liver tumor formation in mice. Gastroenterology 135: 969-979, 2008.

152. Okamura D, Ohtsuka M, Kimura F, et al: Ezrin expression is associated with hepatocellular carcinoma possibly derived from progenitor cells and early recurrence after surgical resection. Mod Pathol 21: 847-855, 2008

153. Jabari S, Meissnitzer M, Quint K, et al: Cellular plasticity of trans- and dedifferentiation markers in human hepatoma cells in vitro and in vivo. Int J Oncol 35: 69-80, 2009.

154. Yu J, Vodyanik MA, Smuga-Otto K, et al: Induced pluripotent stem cell lines derived from human somatic cells. Science 318: 1917-1920, 2007.

155.Zhong X, Li N, Liang S, Huang Q, Coukos G and Zhang L: Identification of microRNAs regulating reprogramming factor LIN28 in embryonic stem cells and cancer cells. J Biol Chem 285: 41961-41971, 2010.

156. Viswanathan SR, Powers JT, Einhorn W, et al: Lin 28 promotes transformation and is associated with advanced human malignancies. Nat Genet 41: 843-848, 2009.

157. Yang X, Lin X, Zhong X, et al: Double-negative feedback loop between reprogramming factor LIN28 and microRNA let-7 regulates aldehyde dehydrogenase 1-positive cancer stem cells. Cancer Res 70: 9463-9472, 2010.

158. Smith LM, Nesterova A, Ryan MC, et al: CD133/prominin-1 is a potential therapeutic target for antibody-drug conjugates in hepatocellular and gastric cancers. Br J Cancer 99: 100-109, 2008.

159. Wang L, Su W, Liu Z, et al: CD44 antibody-targeted liposomal nanoparticles for molecular imaging and therapy of hepatocellular carcinoma. Biomaterials 33: 5107-5114, 2012

160. Fan ST, Yang ZF, Ho DW, Ng MN, Yu WC and Wong J: Prediction of posthepatectomy recurrence of hepatocellular carcinoma by circulating cancer stem cells: a prospective study. Ann Surg 254: 569-576, 2011. 